\title{
Lumbar stenosis: indications for fusion with and without instrumentation
}

Paul W. Detwiler, M.S., M.D., Frederick F. Marciano, M.D., Ph.D., Randall W. Porter, M.D., and Volker K. H. Sonntag, M.D.

Division of Neurological Surgery, Barrow Neurological Institute, Mercy Healthcare Arizona, Phoenix, Arizona

Although the efficacy of posterior decompression for symptomatic lumbar stenosis that is recalcitrant to conservative therapy is well proven, uniform agreement on the need for simultaneous arthrodesis is lacking. The variability in the rate of lumbar fusion with and without instrumentation has been attributed to a number of factors: advances in surgical technique; rapid development of instrumentation; radiographic advances in the diagnosis of disease entities of the lumbar spine; evolution in our understanding of bone healing; improved pre- and postoperative care; aggressive rehabilitation; patient compensation; hospital and surgeon reimbursement; better education of residents, fellows, and practicing neurosurgeons; and, most important, the lack of clear indications based on defined diagnostic categories. Based on review of the literature and their experience at the Barrow Neurological Institute, the authors have attempted to define indications for lumbar fusion with or without instrumentation based on defined diagnostic categories. Clear indications for fusion include trauma, tumor, or infection with two- or three-column injury, iatrogenic instability, and isthmic spondylolisthesis. Relative indications for fusion include degenerative spondylolisthesis, radiographically proven dynamic instability with pain or neurological findings, adult scoliosis, and mechanical back pain. Fusion is rarely indicated with discectomy, abnormal radiographs without appropriate findings (such as degenerative disc disease), facet joint syndrome, failed back surgery, or stable spinal stenosis.

Key Words * lumbar fusion * instrumentation * indication * instability * lumbar stenosis

The indications for lumbar fusion are controversial.[1,21,22,24,33,35,52,58,61,67,68] Geographical variation in the rate of lumbar fusion has been attributed to a number of factors, including technological advances in instrumentation, improved radiographic imaging, evolution of our understanding of bone healing, improved pre- and postoperative care, aggressive rehabilitation, patient compensation, surgeon reimbursement, and the lack of clearly defined indications for fusion.[33,35] The latter is probably the most important factor leading to variable rates of lumbar fusion. In 1995, Katz and coworkers[34] retrospectively reviewed 194 patients who had undergone lumbar decompression for all forms of lumbar stenosis. At a 6-month follow-up examination, $22 \%$ were dissatisfied with their surgical outcome. The authors used these data to reemphasize the need for better criteria for surgical selection. Although 
extensive literature on lumbar fusion has been generated, few prospective studies have evaluated clearly defined diagnostic subgroups.

The rate of lumbar fusion doubled between 1979 and 1990.[33] Understandably, the largest increase occurred in the elderly population. Each year in this country more than 40,000 lumbar fusion procedures, which make up approximately one-fifth of all low-back operations, are performed. Approximately half of the lumbar fusions are combined with placement of instrumentation. As well as increasing the complication rate and the percentage of patients discharged to nursing homes, the addition of fusion to routine decompression increases the hospital and surgical costs by approximately 50\%.[33] The addition of instrumentation to the procedure increases the total cost by approximately $100 \%$.

Franklin and colleagues[15] evaluated the outcome of lumbar fusion in patients receiving Workers' Compensation in Washington state. Overall, the outcome of patients undergoing lumbar fusion with and without instrumentation was worse than outcomes published in the literature. Sixty-eight percent of patients reported that their back or leg pain was worse, and $56 \%$ reported that overall quality of life had not improved or was worse. However, these percentages must be interpreted cautiously. Ironically, $62 \%$ of the patients responded that they would undergo the surgery again. Most of these patients had returned to work and no longer received Workers' Compensation. Psychological factors can also undermine outcome statistics.[3,10,19,62]

A number of variables have been used to evaluate the efficacy of lumbar fusion. In our opinion, comprehensive postoperative assessment must include a thorough and long-term evaluation of radiographically confirmed fusion, complications, pain, function, and patient satisfaction. There is a consensus among most spine surgeons that the outcome of lumbar fusion can be improved with simultaneous progress on a number of fronts. Prospective studies with clearly defined diagnostic categories would probably produce the greatest improvement.[21,24,58,61,67,68] In addition, basic scientific research should address the biomechanics of fusion, the biology of bone healing, and the pathophysiological mechanisms of degenerative bone disease.[4-6,22,52]

\section{EVOLUTION OF UNDERSTANDING}

The development of spinal fusion is attributed to the separate work of Doctors Fred Albee and Russell Hibbs, published in 1911. Their original work involved the treatment of tuberculosis of the spine but was quickly applied to the treatment of trauma, scoliosis, and tumors. During the past 50 years, fusion has been used to treat degenerative disorders. The use of instrumentation to augment lumbar fusion is relatively recent and has generated controversy over the use of anterior fusion versus posterior fusion as well as the use of both.[9,12,16,37,44,48,54,55,60]

This controversy is typified by the recent report of Suk and coworkers.[60] They described 76 patients who underwent posterolateral fusion with pedicle screws with (36 patients) and without (40 patients) interbody fusion after decompression for spondylolitic spondylolisthesis. The mean follow-up period was 2 years. The rate of nonfusion was $8 \%$ in the posterolateral fusion with pedicle screw instrumentation only group and $0 \%$ in the posterolateral fusion with pedicle screw instrumentation group with interbody fusion. The reduction in slippage was $28 \%$ and $42 \%$ and the patient satisfaction rating was $95 \%$ and $97 \%$, respectively. Do these data justify the extra cost and risk of adding interbody fusion? Further evaluation of the data, however, reveals that the percentage of patients with an excellent outcome increased from $45 \%$ to $75 \%$ with the addition of interbody fusion. 


\section{INSTABILITY OF THE SPINE}

Most spine surgeons agree that segmental spinal instability can produce neurological symptoms such as motor weakness, sensory loss, and pain. However, consensus has not been reached about the definition of spinal instability and how to apply the concept to clinical practice. White and Panjabi[63] have defined clinical instability in the spine as "the loss of the ability of the spine under physiological loads to maintain relationships between vertebra in such a way that there is neither damage or subsequent irritation to the spinal cord or nerve roots, and, in addition, there is not development of incapacitating deformity or pain from structural changes."

Kirkaldy-Willis and Farfan[36] have defined spinal instability as "the clinical status of the patient with back problems who with the least provocation steps from the mildly asymptomatic to the severe episodic." They further define the spectrum of clinical manifestations of degenerative spine disease with three contiguous stages: 1) temporary dysfunction, 2) unstable phase, and 3) stabilization.

Based on clinical observations, analysis of pathological material, and review of the literature, Frymoyer and Selby[20] have defined a classification scheme in which there are four types of spinal instability. Type 1 is defined as axial rotational instability. Farfan and Kirkaldy-Willis[13] reported a fixed rotary deformity in some patients who present with low-back pain on twisting about the spine. Plain radiographs revealed narrowing of the disc space, facet degeneration, malalignment of the spinous processes, and a rotational deformity of the pedicles. Type 2, or translational instability,[41] manifests as episodes of recurrent back pain associated with forward translation at the affected level. On plain radiographs, the disc space is narrowed, but the alignment of the spinous processes and pedicles is normal. Lateral flexion-extension radiographs show angulatory collapse of the disc space and forward subluxation at the affected level. This type of instability typically affects women and is most common at L4-5. Type 3, or retrograde spondylolisthetic instability, is most common at L5-S1 and affects as many as one-third of the patients with low-back pain. Plain lateral radiographs demonstrate posterior translation, collapse of the disc space, and facet subluxation. Radiographic imaging of the neural canal often shows lateral stenosis of the spinal canal. Type 4, or postoperative instability, occurs after aggressive decompressive surgery of the lateral spinal canal. Removal of an entire facet, pars interarticularis, or half of each facet at the same level may produce instability. Lateral radiographs demonstrate anterior spondylolisthesis of the affected level compared with preoperative films.

Many investigators have attempted to define instability solely by using radiological methods. Dynamic imaging of the spine with flexion-extension lateral views has been used most often. In general, anterior spondylolisthesis greater than 3 to $4 \mathrm{~mm}$ suggests spinal instability.[18,45,51,59]

Posner and colleagues[53] used a cadaveric model to assess the normal range of motion in lumbar spines subjected to physiological flexion-extension movements. These data were then used to identify the degree of angulation or translation indicative of instability. Panjabi[49] furthered the conceptual framework of biomechanical stability of the lumbar spine by defining an experimental design based on $6 \check{s}$ of freedom: $3 \check{s}$ of translation in the $\mathrm{x}, \mathrm{y}$, and $\mathrm{z}$ coordinate system and 3š of rotation in the same coordinate system. This experimental design can be used to assess the strength, fatigue, and stability of fusion and instrumentation protocols for different types of spinal instability.

White and Panjabi[63] use a point-value checklist to define clinical instability in the lumbar spine. The first category is used to assess the structural and functional integrity of the anterior and posterior elements. The second category is used to assess flexion-extension and resting radiographs; translation 
greater than $4.5 \mathrm{~mm}$ in the sagittal plane on flexion-extension films or rotation greater than $20 \mathrm{~s}$ in the sagittal plane at L4-5 or 25š at L5-S1 is indicative of instability. The third category is used to assess damage to the cauda equina. Finally, the fourth category is used to consider the potential for dangerous loading of the spine. Points are assessed for each of the four categories, and if the total reaches five, the spine is considered to be unstable.

\section{LUMBAR STENOSIS}

At the Barrow Neurological Institute, 206 patients with symptomatic lumbar stenosis were treated initially by decompressive surgery.[50] After a mean follow-up period of 28 months, six patients required fusion procedures. The possible common denominator for these patients was the presence of Grade 2 spondylolisthesis. Consequently, fusion is not routinely combined with lumbar decompression unless Grade 2 or worse spondylolisthesis is coexistent.

\section{ISTHMIC SPONDYLOLISTHESIS}

Patients with isthmic spondylolisthesis typically present during their teenage years. At diagnosis, the most important prognostic indicators for future low-back pain are the patient's age and the degree of slippage.[57] When the degree of spondylolisthesis is greater than $25 \%$ of the vertebral body width, the risk for developing back pain increases. The incidence of isthmic spondylolisthesis reported in autopsy studies is approximately $5 \%$.

Wiltse, et al.,[64] presented a classification system for spondylolysis and spondylolisthesis. Type 1 (dysplastic lesion) is a congenital abnormality of the upper sacrum or arch of L-5 that allows spondylolisthesis. Type 2, which is discussed in this section, is called isthmic and defines a lesion in the pars interarticularis. Three categories of Type 2 lesions are recognized: 1) lytic-fatigue fracture of the pars, 2) elongated but intact pars, and 3) acute fracture. Type 3 (degenerative spondylolisthesis) is related to long-standing intersegmental instability and is discussed in the next section. Type 4 (traumatic) is related to fractures that are not located in the pars interarticularis. Pathological Type 4 spondylolisthesis can be caused by a generalized or localized bone disorder.

Approximately half of the patients with spondylolysis progress to spondylolisthesis. Some authors have recommended repairing the pars interarticularis defect in spondylolysis or Grade 1 spondylolisthesis. In general, an L5-S1 fusion has been used to treat Grades 1 and 2 spondylolisthesis, and an L4-S1 fusion has been used to treat higher grades of spondylolisthesis.

The indications for lumbar arthrodesis in children and adolescents with spondylolisthesis are controversial. The potential risk of aggressive spondylolisthesis has sometimes been considered an indication for surgery. The treatment of adolescents with moderate-to-severe spondylolisthesis without associated signs or symptoms is also controversial. Both Hensinger[28] and Laurent and Österman[38] recommend that all adolescents with Grade 3 or 4 spondylolisthesis undergo fusion, regardless of their symptomatology. Fusion in this group is also supported by the improved long-term prognosis when arthrodesis is performed in childhood rather than adulthood.[25] Conversely, however, some long-term follow-up studies support conservative treatment of asymptomatic children and teenagers.[2,17] We believe that fusion is indicated when 1) mechanical low-back pain persists or progresses or neurological symptoms are recalcitrant to conservative therapy, 2) spondylolisthesis is symptomatic and high grade, and 3) a child or adolescent has progressive symptoms and spondylolisthesis greater than Grade 1.

Attempted reduction of isthmic spondylolisthesis is associated with a high rate of neurological 
complications and instrumentation failure. The reported rate of transient or permanent neurological sequelae has been approximately $30 \%$. Further fueling the controversy about the appropriate surgical procedure is the issue of when to perform decompressive surgery. The literature on this topic is inconclusive.

Hanley and Levy[25] reported on 50 consecutive patients with isthmic lumbosacral spondylolisthesis. Twenty-two patients who experienced mechanical low-back pain without radicular symptoms were treated by lateral fusion of L-4 to the sacrum with autologous iliac crest bone grafting. Twenty-eight patients with both back pain and radicular symptoms underwent neural decompression and lateral fusion from L-4 to the sacrum and iliac crest bone grafting combined with the Gill procedure. No instrumentation was used. At their 40-month follow-up examination, 50\% of patients in both groups reported an excellent or good outcome. This percentage did not correlate with the degree of vertebral body slippage. A trend toward unsatisfactory outcomes was present among males, middle-aged individuals, individuals who smoked cigarettes, and patients with radicular symptoms. Harris and Weinstein[27] have addressed long-term outcomes in patients with Grade 3 or 4 spondylolisthesis treated conservatively (11 patients) and surgically (21 patients). Patients in the surgical group underwent posterior interlaminar fusion. At a mean 18-year follow up in the conservatively treated group, $36 \%$ were asymptomatic, $55 \%$ had mild symptoms, and one patient had significant symptoms. Forty-five percent of patients had an abnormal finding on neurological examination, but none was incontinent. All of the patients in this group led an active life. At a mean 24-year follow up in the surgical group, 57\% were asymptomatic, $38 \%$ had mild symptoms, and one had significant symptoms. Neurological examination revealed a significant finding in half of these patients. Seventy-five percent of the patients in the surgical group reported that their symptoms had improved over time compared with $36 \%$ in the conservatively treated group. The degree of spondylolisthesis was not related to outcome in either group. Radiographic measurements of the percentage and angle of slippage did not predict outcome in either group.

Johnson and Kirwan[30] followed 17 patients in whom posterior or posterolateral fusion was performed for severe spondylolisthesis. At a mean 14-year follow up, seven patients experienced occasional back pain. Nine patients believed that their spinal deformity had improved, and 16 patients rated their surgical outcome as excellent.

Boxall, et al.,[7] retrospectively reviewed 43 patients with Grade 3 or greater spondylolisthesis at L5-S1. Two patients were asymptomatic at presentation, whereas 41 patients had symptoms of back pain, radicular pain, hamstring tightness, or gait disturbance. Treatment groups were as follows: four patients were treated conservatively, 11 with fusion, 18 with decompression and arthrodesis, and 10 with reduction and fusion. At a mean follow up of 6.7 years, $3 \%$ of the patients were fully active without symptoms, and $14 \%$ were fully active without limitations but experienced occasional mild pain. Of the patients with a solid arthrodesis, spondylolisthesis progressed in $26 \%$. Nine patients had a significant increase in the angle of slippage. Of nine patients with a pseudarthrosis, five experienced progressive slipping. Kaneda and colleagues[32] performed posterolateral fusion with distraction-rod instrumentation in 53 cases of isthmic spondylolisthesis. At 3-year follow-up review, 71\% had excellent, $21 \%$ had good, $6 \%$ had fair, and the remainder had unsatisfactory outcomes.

\section{DEGENERATIVE SPONDYLOLISTHESIS}

Degenerative spondylolisthesis (Frymoyer type 3)[20] is an acquired condition in which disc degeneration is believed to lead to facet arthropathy and segmental instability. It is seen most frequently 
in elderly patients presenting with low-back pain and radicular symptoms or neurogenic claudication, whose neurological examination is often unremarkable. Radiographic evaluation demonstrates anterior spondylolisthesis, usually at L4-5 and, less frequently, at L3-4. Typically, the degree of slippage is approximately one-fourth of the vertebral body width. Dynamic lateral flexion-extension radiographs can be used to document gross instability.

The use of fusion to treat degenerative spondylolisthesis continues to be controversial.[11,14,40,46,56] Feffer, et al.,[14] retrospectively compared degenerative spondylolisthesis in eight patients who had undergone lumbar decompression and fusion (type of fusion not reported) with 11 patients who had undergone only decompression. Preoperatively, all patients experienced leg and back pain. Radiographic studies confirmed thecal sac compression and facet joint arthrosis with anterior spondylolisthesis of at least $3 \mathrm{~mm}$. The follow-up period for the fusion group was 42 months. All patients in this group were satisfied with their surgical outcomes. No spinal instability was documented on lateral flexion-extension radiographs. Among the decompression only group, with a mean follow-up period of 25 months, five patients achieved good, three patients had fair, and three patients had poor outcomes. Four patients developed radiographically confirmed instability. The degree of instability, type of instability, and surgical outcome were correlated.

For patients with degenerative spondylolisthesis at L4-5, DePalma and Rothman[11] have recommended complete decompression of the neural elements combined with intertransverse process fusion from L-4 to L-5. Of their patients, $64 \%$ reported total pain relief, whereas $20 \%$ experienced partial relief. Reynolds and Wiltse[56] reported a patient satisfaction level of $78 \%$ for those who underwent facet-sparing procedures. In contrast, the level of patient satisfaction was only $33 \%$ among those who had undergone complete removal of a facet.

Johnsson and coworkers[31] studied postoperative spinal instability in 45 patients undergoing decompression for lumbar stenosis. Preoperatively, 20 patients had degenerative spondylolisthesis and 25 had acquired spinal stenosis. Postoperative slippage was seen in 18 patients. The risk of further slippage was higher for the degenerative spondylolisthesis group but it did not influence operative outcome.

Lombardi and colleagues[40] retrospectively reviewed 107 patients who were treated for degenerative spondylolisthesis with one of three surgical protocols. No correlation was found between the degree of preoperative spondylolisthesis and surgical outcome. Follow-up periods ranged from 2 to 7 years. Among patients treated with decompressive laminectomy and aggressive facetectomy, 33\% had a good-to-excellent outcome. Among patients treated with laminectomy and medial facetectomy, $80 \%$ had a good-to-excellent outcome. The third group, treated using a protocol similar to that of the second group, also underwent intertransverse process fusion between slipped levels. Among these patients, 90\% had a good-to-excellent outcome.

Nasca[46,47] studied the outcome of surgical decompression with fusion in patients with lumbar stenosis from multiple causes. He concluded that fusion was indicated in patients undergoing decompression for stenosis with degenerative spondylolisthesis, facet joint degeneration, degenerative disc disease with instability, and scoliosis with instability. Lehmann, et al.,[39] performed a retrospective, 33-year follow-up study in 62 patients who had undergone lumbar fusion for radiographically confirmed instability, intraoperative instability, degenerative disc disease, facet joint syndrome, spondylosis, degenerative spondylolisthesis, and chronic low-back pain. Of 33 patients available for physical examination, spinal instability was found to be present in 15 (45\%), and 14 (42\%) had recurrent stenosis. 
The degree of spondylolisthesis and stenosis did not correlate with the patients' symptoms at follow up. The best long-term outcome was reported in patients who had undergone decompression and fusion for spondylolisthesis.

Herkowitz and Kurz[29] retrospectively studied the benefit of routine arthrodesis for degenerative lumbar spondylolisthesis and stenosis. Fifty patients were equally divided into a nonfusion group and an arthrodesis group. At a mean 3-year follow-up time, the level of patient satisfaction was significantly higher in the arthrodesis group. Twenty-four patients reported a satisfactory outcome, and only one was unsatisfied. In the nonfusion group, 11 patients were satisfied with their outcome and 14 were not.

The simultaneous use of instrumentation for treating lumbar instability is also controversial. Hanley[23] and Harrington and Dickson[26] advocate the use of instrumentation to correct spinal deformities and thereby to decrease tension on neural elements. Markwalder[43] found that $91 \%$ of 100 patients achieved excellent outcomes when instrumentation was combined with fusion to treat degenerative spondylolisthesis. The results from a prospective, randomized study of 124 patients undergoing lumbar or lumbosacral fusion for degenerative conditions also support the use of instrumentation.[66] The diagnostic groups included isthmic spondylolisthesis, degenerative spondylolisthesis, degenerative disc disease, degenerative scoliosis, spinal stenosis, and failed back surgery. The rate of successful fusion for patients undergoing posterolateral fusion with autogenous bone graft was $65 \%$, and $71 \%$ achieved a good-to-excellent outcome. The rate of successful fusion increased to $77 \%$ in patients undergoing posterolateral fusion supplemented by a semirigid pedicle screw/plate fixation system, and $89 \%$ had a satisfactory outcome. The rate of successful fusion further increased to $95 \%$ in patients undergoing fusion with a rigid pedicle screw/rod fixation system, and 95\% experienced a good-to-excellent outcome.

To define the role of fusion and instrumentation further, Bridwell, et al.,[8] prospectively studied 44 patients with degenerative spondylolisthesis and stenosis. Patients were randomized to one of three groups: nonfusion (nine patients), transverse process fusion with autogenous iliac bone graft without instrumentation (10 patients), and transverse process fusion with autogenous iliac crest bone graft and instrumentation (24 patients), with a mean follow-up period of 3 years. Fusion with instrumentation significantly improved the rate of successful fusion. Furthermore, patients in the first two groups had a higher rate of postoperative spondylolisthesis: four of the nine patients in the nonfusion group and seven of the 10 patients who underwent fusion without instrumentation. Only one of the 24 patients in whom instrumentation was placed had progressive spondylolisthesis, which was defined as angulation of the motion segment greater than 10š or translation of the vertebral body greater than $3 \mathrm{~mm}$ on lateral flexion-extension radiographs.

Yuan, et al.,[65] studied a historical cohort of patients who underwent fusion with pedicle screw fixation of the thoracic, lumbar, and sacral spine for the treatment of degenerative spondylolisthesis or fractures. Of 2633 patients undergoing surgery for degenerative spondylolisthesis, 2177 patients were treated with and 456 patients were treated without pedicle screws. The use of pedicle screws was safe and improved the patients' pain, fusion success rate, and neurological and functional outcomes.

Mardjetko, et al.,[42] performed a metaanalysis of 25 published studies to assess the advantages of instrumentation in the treatment of degenerative lumbar spondylolisthesis. An overall satisfaction rating of $69 \%$ was calculated for the studies in which decompression without fusion had been evaluated. Fusion without instrumentation increased the satisfaction rating to $90 \%$. The average rate of successful fusion was $86 \%$. The level of patient satisfaction with decompression and fusion with pedicle screws was $86 \%$, 
the rate of fusion was $93 \%$, and the complication rate was $10 \%$. They concluded that their findings supported the use of fusion in the management of degenerative lumbar spondylolisthesis. Overall, however, it is difficult to assess the advantages of instrumentation because the study designs and treatment protocols in the literature are not uniform.

\section{CONCLUSIONS}

Based on review of the literature and our own experience at the Barrow Neurological Institute, we have attempted to define indications for lumbar fusion, with or without instrumentation, based on defined diagnostic categories. Clear indications for fusion include trauma, tumor, or infection with two- or three-column injury, iatrogenic instability, and isthmic spondylolisthesis. Relative indications include degenerative spondylolisthesis, radiographically proven dynamic instability with pain or neurological findings, adult scoliosis, and mechanical back pain. Fusion is rarely indicated in the setting of routine discectomy, abnormal radiographs without appropriate findings (such as degenerative disc disease), facet joint syndrome, failed back surgery, or stable spinal stenosis.

\section{References}

1. Andersson GB, Weinstein JN: Introduction to focus issue on fusion. Spine 20 (Suppl 24):75S, 1995

2. Apel DM, Lorenz MA, Zindrick MR: Symptomatic spondylolisthesis in adults: four decades later. Spine 14:345-348, 1989

3. Blumenthal SL, Baker J, Dossett A, et al: The role of anterior lumbar fusion for internal disc disruption. Spine 13:566-569, 1988

4. Boden SD, Schimandle JH: Biologic enhancement of spinal fusion. Spine 20 (Suppl 24):113S-123S, 1995

5. Boden SD, Sumner DR: Biologic factors affecting spinal fusion and bone regeneration. Spine 20 (Suppl 24):102S-112S, 1995

6. Boden SD, Sumner DR, Andersson GBJ, et al: Biologic issues in lumbar spinal fusion. 1995 Focus Issue Meeting on Fusion. Spine 20 (Suppl 24):100S-101S, 1995

7. Boxall D, Bradford DS, Winter RB, et al: Management of severe spondylolisthesis in children and adolescents. J Bone Joint Surg (Am) 61:479-495, 1979

8. Bridwell KH, Sedgewick TA, O'Brien MF, et al: The role of fusion and instrumentation in the treatment of degenerative spondylolisthesis with spinal stenosis. J Spinal Disord 6:461-472, 1993

9. Carl AL, Tranmer BI, Sachs BL: Anterolateral dynamized instrumentation and fusion for unstable thoracolumbar and lumbar burst fractures. Spine 22:686-690, 1997

10. Crock HV: Internal disc disruption. A challenge to disc prolapse fifty years on. Spine 11:650-653, 1986

11. DePalma AF, Rothman RH: Surgery of the lumbar spine. Clin Orthop 63:162-170, 1969

12. Dick JC, Brodke DS, Zdeblick TA, et al: Anterior intrumentation of the thoracolumbar spine. A biomechanical comparison. Spine 22:744-750, 1997 
13. Farfan HF, Kirkaldy-Willis WH: The present status of spinal fusion in the treatment of lumbar intervertebral joint disorders. Clin Orthop 158:198-214, 1981

14. Feffer HL, Wiesel SW, Cuckler JM, et al: Degenerative spondylolisthesis. To fuse or not to fuse. Spine 10:287-289, 1985

15. Franklin GM, Haug J, Heyer NJ, et al: Outcome of lumbar fusion in Washington State workers' compensation. Spine 19:1897-1904, 1994

16. Fraser RD: Interbody, posterior, and combined lumbar fusions. Spine 20 (Suppl 24):167S-177S, 1995

17. Fredrickson BE, Baker D, McHolick WJ, et al: The natural history of spondylolysis and spondylolisthesis. J Bone Joint Surg (Am) 66:699-707, 1984

18. Frymoyer JW: Low back pain. The role of spine fusion. Neurosurg Clin North Am 2:933-954, 1991

19. Frymoyer JW, Hanley E, Howe J, et al: Disc excision and spine fusion in the management of lumbar disc disease. A minimum ten-year followup. Spine 3:1-6, 1978

20. Frymoyer JW, Selby DK: Segmental instability. Rationale for treatment. Spine 10:280-286, 1985

21. Garfin SR, Spratt KF, Andersson GBJ, et al: Use of internal fixation instrumentation. Introduction. 1995 Focus Issue Meeting on Fusion. Spine 20 (Suppl 24):154S-156S, 1995

22. Goel VK, Pope MH: Biomechanics of fusion and stabilization. Spine 20 (Suppl 24):S85-S99, 1995

23. Hanley EN Jr: Decompression and distraction-derotation arthrodesis for degenerative spondylolisthesis. Spine 11:269-276, 1986

24. Hanley EN Jr: The indications for lumbar spinal fusion with and without instrumentation. Spine 20 (Suppl 24):143S-153S, 1995

25. Hanley EN Jr, Levy JA: Surgical treatment of isthmic lumbosacral spondylolisthesis. Analysis of variables influencing results. Spine 14:48-50, 1989

26. Harrington PR, Dickson JH: Spinal instrumentation in the treatment of severe progressive spondylolisthesis. Clin Orthop 117:157-163, 1976

27. Harris IE, Weinstein SL: Long-term follow-up of patients with grade-III and IV spondylolisthesis. Treatment with and without posterior fusion. J Bone Joint Surg (Am) 69:960-969, 1987

28. Hensinger RN: Current concepts review. Spondylolysis and spondylolisthesis in children and adolescents. J Bone Joint Surg (Am) 71:1098-1107, 1989

29. Herkowitz HN, Kurz LT: Degenerative lumbar spondylolisthesis with spinal stenosis. A prospective study comparing decompression with decompression and intertransverse process arthrodesis. J Bone Joint Surg (Am) 73:802-808, 1991

30. Johnson JR, Kirwan EOG: The long-term results of fusion in situ for severe spondylolisthesis. J Bone Joint Surg (Br) 65:43-46, 1983 
31. Johnsson KE, Willner S, Johnsson K: Postoperative instability after decompression for lumbar spinal stenosis. Spine 11:107-110, 1986

32. Kaneda K, Satoh S, Nohara Y, et al: Distraction rod instrumentation with posterolateral fusion in isthmic spondylolisthesis. 53 cases followed for 18-89 months. Spine 4:383-389, 1985

33. Katz JN: Lumbar spinal fusion. Surgical rates, costs, and complications. Spine 20 (Suppl 24):78S-83S, 1995

34. Katz JN, Lipson SJ, Brick GW, et al: Clinical correlates of patient satisfaction after laminectomy for degenerative lumbar spinal stenosis. Spine 20:1155-1160, 1995

35. Katz JN, Spratt KF, Andersson GB, et al: Epidemiology introduction. 1995 Focus Issue Meeting on Fusion. Spine 20 (Suppl 24):76S-77S, 1995

36. Kirkaldy-Willis WH, Farfan HF: Instability of the lumbar spine. Clin Orthop 165:110-123, 1982

37. Kozak JA, Heilman AE, O'Brien JP: Anterior lumbar fusion options. Technique and graft materials. Clin Orthop 300:45-51, 1994

38. Laurent LE, Österman K: Operative treatment of spondylolisthesis in young patients. Clin Orthop 117:85-91, 1976

39. Lehmann TR, Spratt KF, Tozzi JE, et al: Long-term follow-up of lower lumbar fusion patients. Spine 12:97-104, 1987

40. Lombardi JS, Wiltse LL, Reynolds J, et al: Treatment of degenerative spondylolisthesis. Spine 10:821-827, 1985

41. Macnab I: The traction spur. An indicator of segmental instability. J Bone Joint Surg (Am) 53:663-670, 1971

42. Mardjetko SM, Connolly PJ, Shott S: Degenerative lumbar spondylolisthesis. A meta-analysis of literature 1970-1993. Spine 19 (Suppl 20):2256S-2265S, 1994

43. Markwalder TM: Surgical management of neurogenic claudication in 100 patients with lumbar spinal stenosis due to degenerative spondylolisthesis. Acta Neurochir 120:136-142, 1993

44. Mayer HM: A new microsurgical technique for minimally invasive anterior lumbar interbody fusion. Spine 22:691-700, 1997

45. Mirkovic S, Garfin SR: "Segmental" spinal instability as related to the degenerative disc. Semin Spine Surg 3:119-123, 1991

46. Nasca RJ: Rationale for spinal fusion in lumbar spinal stenosis. Spine 14:451-454, 1989

47. Nasca RJ: Surgical management of lumbar spinal stenosis. Spine 12:809-816, 1987

48. O'Brien JP, Dawson MHO, Heard CW, et al: Simultaneous combined anterior and posterior fusion. A surgical solution for failed spinal surgery with a brief review of the first 150 patients. Clin Orthop 203:191-195, 1986 
49. Panjabi MM: Biomechanical evaluation of spinal fixation devices: I. A conceptual framework. Spine 13:1129-1134, 1988

50. Pappas CTE, Sonntag VKH: Lumbar stenosis in the elderly. Neurosurg Q 4:102-112, 1994

51. Penning L, Blickman JR: Instability in lumbar spondylolisthesis: a radiologic study of several concepts. AJR 134:293-301, 1980

52. Pope MH, Goel VK, Sumner DR, et al: Biomechanics introduction. 1995 Focus Issue Meeting on Fusion. Spine 20 (Suppl 24):84S, 1995

53. Posner I, White AA III, Edwards WT, et al: A biomechanical analysis of the clinical stability of the lumbar and lumbosacral spine. Spine 7:374-389, 1982

54. Ray CD: Threaded fusion cages for lumbar interbody fusions. An economic comparison with 360š fusions. Spine 22:681-685, 1997

55. Ray CD: Threaded titanium cages for lumbar interbody fusions. Spine 22:667-680, 1997

56. Reynolds JB, Wiltse LL: Surgical treatment of degenerative spondylolisthesis. Spine 4:148-149, 1979 (Abstract)

57. Rosenberg NJ: Degenerative spondylolisthesis. Predisposing factors. J Bone Joint Surg (Am) 57:467-474, 1975

58. Sonntag VKH, Marciano FF: Is fusion indicated for lumbar spinal disorders? Spine 20 (Suppl 24):138S-142S, 1995

59. Stokes IA, Frymoyer JW: Segmental motion and instability. Spine 12:688-691, 1987

60. Suk SI, Lee CK, Kim WJ, et al: Adding posterior lumbar interbody fusion to pedicle screw fixation and posterolateral fusion after decompression in spondylolytic spondylolisthesis. Spine 22:210-220, 1997

61. Vaccaro AR, Garfin SR: Internal fixation (pedicle screw fixation) for fusions of the lumbar spine. Spine 20 (Suppl 24):157S-165S, 1995

62. Weatherley CR, Prickett CF, O'Brien JP: Discogenic pain persisting despite solid posterior fusion. J Bone Joint Surg (Br) 68: 142-143, 1986

63. White AA III, Panjabi MM: Clinical Biomechanics of the Spine, ed 2. Philadelphia: JB Lippincott, 1990, pp 342-378

64. Wiltse LL, Newman PH, Macnab I: Classification of spondylolisis and spondylolisthesis. Clin Orthop 117:23-29, 1976

65. Yuan HA, Garfin SR, Dickman CA, et al: A historical cohort study of pedicle screw fixation in thoracic, lumbar, and sacral spinal fusions. Spine 19 (Suppl 20):2279S-2296S, 1994

66. Zdeblick TA: A prospective, randomized study of lumbar fusion. Preliminary results. Spine 18:983-991, 1993 
67. Zdeblick TA: The treatment of degenerative lumbar disorders. A critical review of the literature. Spine 20 (Suppl 24):126S-137S, 1995

68. Zdeblick TA, Hanley EN Jr, Sonntag VK, et al: Indications for lumbar spinal fusion. 1995 Focus Issue Meeting on Fusion. Spine 20 (Suppl 24):124S-125S, 1995

Manuscript received July 1, 1997.

Accepted in final form July 15, 1997.

Address reprint requests to: Volker K. H. Sonntag, M.D., c/o Neuroscience Publications, Barrow

Neurological Institute, 350 West Thomas Road, Phoenix, Arizona 85013-4496. email:

neuropub@mha.chw.edu 